\title{
ANALYSIS OF A MAGNETICALLY TUNABLE PATCH RADIATOR INCLUDING METALLIZATION AND FERRITE-SUBSTRATE LOSSES
}

Jens Bornemann* and Zhenglian Cai

Laboratory for Lightwave Electronics, Microwaves and Communications (L.LiMiC)

Centre for Advanced Materials and Related Technology

(CAMTEC)

University of Victoria, Victoria B.C. Canada V8W 3P6

\section{INTRODUCTION}

With the development of ferrite thin-film technology and possible monolithic integration of such substrates [1], microstrip radiators on ferrite substrates [2], [3] become increasingly attractive in tunable patch antenna configurations. For the accurate prediction of circuit performance, it is necessary - as in, e.g., GaAs-based MMIC applications - that theoretical models include such material characteristics as substrate losses and anisotropies, finite metallization thickness and finite conductivity of the radiating patch and the ground metallization. While substrate losses are sometimes considered in patch antenna models, the metallizations are still assumed ideal and of vanishing thickness. That this assumption can result in large errors is well known from investigations carried out for MMIC structures, e.g., [4].

Therefore, this paper focuses on the incorporation of conductor, bottom metallization and substrate losses in the analysis of magnetically tunable patch radiators on ferrite substrates. A modified spectral-domain immittance approach is used to investigate the influence of different magnitudes and directions of extemal magnetic bias on the resonant frequency and Q-factor. Pattern calculations demonstrate that the basic shape of radiation characteristics is maintained when applying external magnetic bias.

\section{THEORY}

Fig. 1 shows the patch antenna geometry and indicates the substrate losses as well as those of the conducting parch and bottom metallization. The ferrite material is represented by tensors

$$
\langle\vec{\mu}\rangle=\mu_{o}\left[\begin{array}{ccc}
\mu_{x} & 0 & 0 \\
0 & \mu & j \kappa \\
0 & -j \kappa & \mu
\end{array}\right] \quad\langle\vec{\mu}\rangle=\mu_{o}\left[\begin{array}{ccc}
\mu & 0 & j \kappa \\
0 & \mu_{y} & 0 \\
-j \kappa & 0 & \mu
\end{array}\right] \quad\langle\vec{\mu}\rangle=\mu_{o}\left[\begin{array}{ccc}
\mu & j \kappa & 0 \\
-j \kappa & \mu & 0 \\
0 & 0 & \mu_{z}
\end{array}\right]
$$

for magnetic bias in $\mathrm{x}-, \mathrm{y}-, \mathrm{z}$-direction, respectively. Although the tensor components can be complex in general, well-known real expressions [5] are used in this investigation while the substrate losses are entirely attributed to the complex permittivity.

$$
\varepsilon_{r c}=\varepsilon_{r}[1-\tan \delta]
$$

The spectral-domain immittance approach (SDIA) [6] is then applied to 
decouple TE-to-y and TM-to-y modes in the spectral domain. After obtaining the related propagation constants and immittances, the finite patch and bottom metallizations are incorporated via their surface inpedances

$$
Z_{p, g}= \begin{cases}\sqrt{\omega \mu_{\mathrm{o}} / 2 \sigma_{p, g}} & t_{p, g} \geq 3 \lambda \\ 1 /\left(t_{p, g} \sigma_{p, g}\right) & t_{p, g}<3 \lambda\end{cases}
$$

where subscripts $p, g$ Iepresent patch, ground metalization, respectively, and $\lambda$ is the penetration depth. While the ground metallization can directly be considered in the SDIA procedure, a complex boundary condition [7] is applied for the conducting patch to modify the Green's dyadic functions.

For details regarding this method and comparison with available data involving ferrite substrates, the reader is referred to [8]. The underlying principles of resonant frequency/Q-factor and pattern calculations are presented in [9], [10], respectively. Computation time for one set of parameters is approximately ten minutes on a workstation compatible with a IBM RS6000/530.

\section{RESULTS}

At the example of a MIC configuration, the set of Fig. 2 shows the effects of external magnetic bias on the resonant frequency, Q-factor and radiation pattern of the patch. For the square geometry investigated, the tuning range is comparable with respect to bias applied in all three directions of the cartesian coordinate system. The ferrite behaves as a lossy dielectric at zero external bias and reduces its effective complex permeability with increasing bias. Consequently, both resonant frequency (Fig. 2 top) and Q-factor (Fig. 2 mid) increase with applied d.c. bias. The increase in Q-factor, i.e. reduction of losscs, with applied bias is also visible in the radiation pattern (Fig. 2 bottom) which is normalized to the maximum field strength calculated in the lossless case. With the varying magnetic bias chosen in the y-direction as example, the basic shape and beamwidth of the patterns are changed only marginally. The corresponding resonant frequencies are those plotted in Fig. 2 (top).

Figs. 3 show a similar investigation with respect to thin-film technology as proposed in [1]. The fundamental differences compared to Figs. 2 are a slightly reduced tuning range and a significantly lower $\mathrm{Q}$-factor. The $\mathrm{Q}$-factor reduction is predominantly due to the lower saturation magnetization assumed in this case. Note that the difference in surface impedance for the conducting patch in both cases (Fig. 2 and Fig. 3) differs only by some milliohms so that the thin conductor and bottom metallization in Fig. 3 do not contribute to the lower Q-factor.

\section{CONCLUSION}

An extended spectral domain immittance approach is used to rigorously analyze rectangular patch radiators on ferrite substrate. The theoretical model includes the effects of a lossy conducting patch, a lossy ground metallization as well as a five-element tensor and a complex permittivity to represent the ferrite substrate. For a square patch geometry, it is demonstrated that tuning range and $Q$ factor are independent of the direction of magnetic bias. Radiation patterns maintain their basic shape and beamwidth during tuning while the efficiency increases with external bias. 


\section{REFERENCES}

[1] P.J. Rainville and F.J. Harackiewicz, "Magnetic tuning of a microstrip patch antenna fabricated on a ferrite film", IEEE Microwave Guided Wave Lett., vol. 2, pp. 483-485, Dec. 1992

[2] D.M. Pozar, "Radiation and scattering characteristics of microstrip antennas on normally biased ferrite substrates", IEEE Trans. Antennas Propagat., vol. AP-40, pp. 1084-1092, Sep. 1992.

[3] S.N. Das and S.K. Chowdhury, "Rectangular microstrip antennas on a ferrite substrate", IEEE Trans. Antennas Propagat., vol. AP-30, pp. 499-502, May 1982

[4] W. Heinrich, "Full-wave analysis of conductor losses on MMIC transmission lines", IEEE Trans. Microwave Theory Tech., vol. 38, pp. 1468-1472, Oct. 1990 .

[5] S. Bolioli, H. Benzina, H. Baudrand and B. Chan, "Centimeter-wave microstrip phase shifter on a ferrite-dielectric substrate", IEEE Trans. Microwave Theory Tech., vol. 37, pp. 698-705, April 1989.

[6] T. Itoh, "Spectral domain immittance approach for dispersion characteristics of generalized printed transmission lines", IEEE Trans. Microwave Theory Tech., vol, MTT-28, pp. 733-736, July 1980.

[7] J.M. Pond, C.M. Krowne and W.L. Carter, "On the application of complex resistive boundary conditions to model transmission lines consisting of very thin superconductors", IEEE Trans. Microwave Theory Tech., vol. 37, pp. 181-190, Jan. 1989.

[8] Z. Cai and J. Bomemann, "Generalized spectral-domain analysis for multilayered complex media and high-Tc superconductor applications", IEEE Trans. Microwave Theory Tech., vol. 40, pp. 2251-2257, Dec. 1992

[9] Z. Cai and J. Bornemann, "Full-wave analysis of high-Tc superconductor patch antenna on lossy bi-anisotropic substrates", in 1992 IEEE-APS Int. Symp. Dig., pp 983-986.

[10] Z. Cai and J. Bornemann, "Loss analysis for the radiation characteristics of rectangular microstrip elements", in 1993 IEEE-APS Int. Symp. Dig., pp. 630-633.

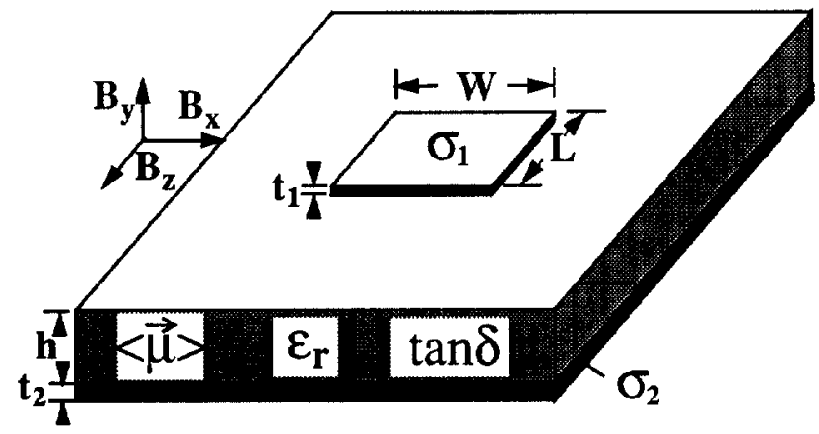

Fis 1 Nonideal microstrip patch radiator on lossy ferrite substrate and imperfect ground metallization. 

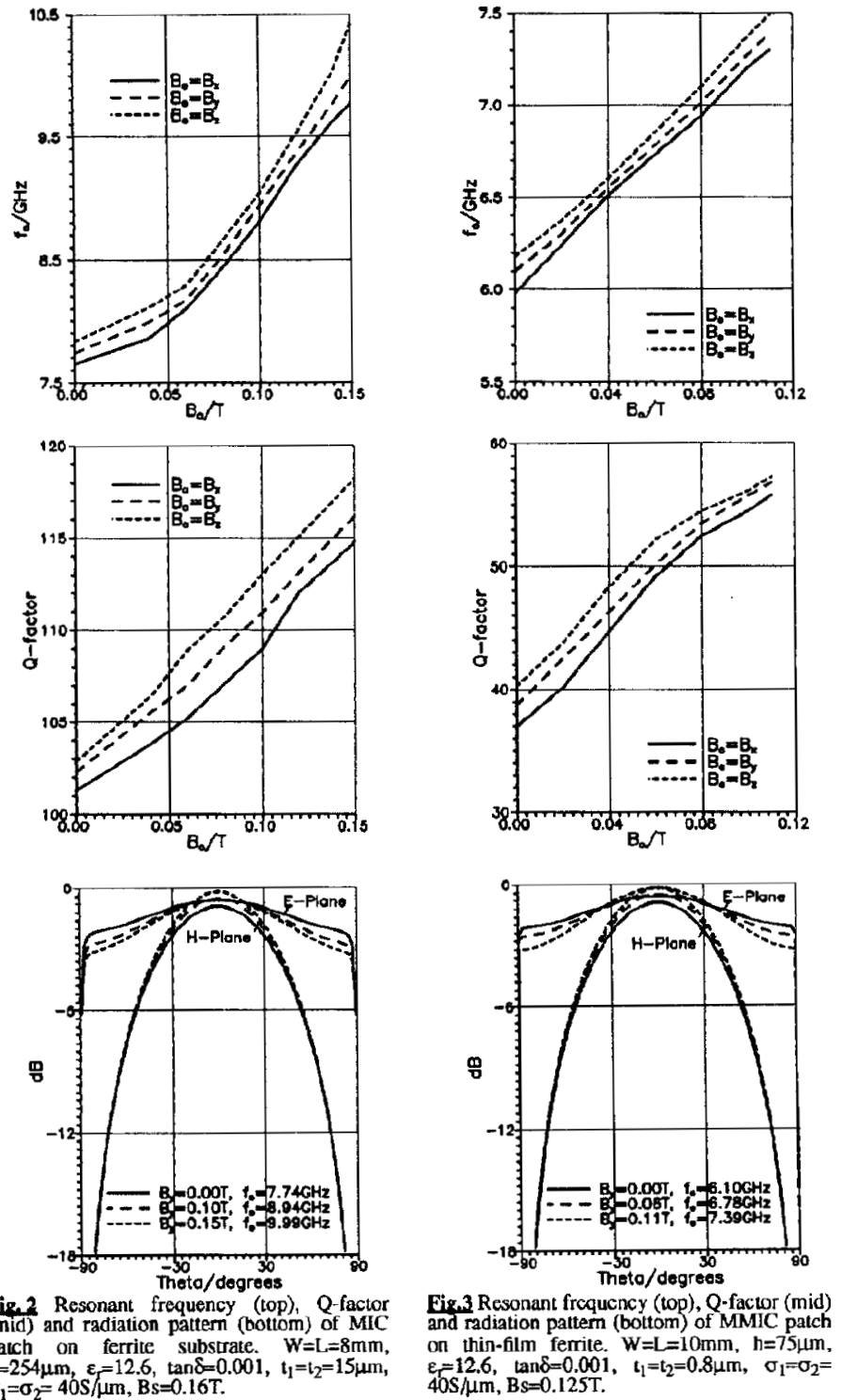